\title{
Long-term histological results of ossicular chain reconstruction using bioceramic implants
}

\author{
HORIA MOCANU ${ }^{1 *}$, ADELA-IOANA MOCANU ${ }^{2}$, ANA MIRUNA DRĂGOI $^{3}$ and MARIAN RĂDULESCU ${ }^{4 *}$ \\ ${ }^{1}$ Department of ENT\&HNS, Faculty of Medicine, Titu Maiorescu University, 031593 Bucharest; ${ }^{2}$ Department of ENT\&HNS, \\ Bucharest Emergency University Hospital, 050098 Bucharest; ${ }^{3}$ Department of Neuropsychiatry, \\ Alexandru Obregia Clinical Hospital of Psychiatry, 041902 Bucharest; ${ }^{4}$ Department of ENT\&HNS, \\ Carol Davila University of Medicine and Pharmacy, 020021 Bucharest, Romania
}

Received September 30, 2020; Accepted October 30, 2020

DOI: $10.3892 /$ etm.2021.9692

\begin{abstract}
Middle ear problems are addressed using tympanoplasty, which requires a mobile tympanic membrane and secure sound-conducting mechanism. The present is a long-term, retrospective, non-controlled study on the results of ossiculoplasty using bioceramic implants of autochthonous origin and which defined the statistical relevant factors that could influence the rate of implant rejection. In a cohort of 108 patients ossiculoplasties with bioceramic implants were performed and patients were followed up clinically for a period of minimum 7 years. Several factors were identified that could influence the results of the implantation and the statistical correlations were studied. The rejection rate after 9.12 years was $21 \%$ (23 patients). Histological integration rate was $79 \%$ (85 patients), similar to results reported in literature for both bioceramic and titanium implants. Hydroxyapatite has many of the ideal characteristics required to be a good prosthesis with a high degree of biocompatibility, very low extrusion rate, low risk of disease transmission and good functional results. Although perhaps considered of historical interest, bioceramic implants are cheaper and can be produced locally, which is a great advantage for struggling economies.
\end{abstract}

\section{Introduction}

The generic term tympanoplasty describes procedures that address the status of the middle ear from the tympanic membrane to the vestibule. A successful tympanoplasty requires a mobile tympanic membrane (TM) and a secure sound-conducting mechanism (1) within the middle ear.

Correspondence to: Dr Horia Mocanu, Department of ENT\&HNS, Faculty of Medicine, Titu Maiorescu University, 67A Gheorghe Petrașcu Street, 031593 Bucharest, Romania

E-mail: horia.mocanu@prof.utm.ro

${ }^{*}$ Contributed equally

Key words: hydroxyapatite, mineralization, prostheses, implants, ossiculoplasty, ceramics
Ossiculoplasty, also known as ossicular chain reconstruction (OCR) is the process of recreating an interrupted ossicular chain and re-establishing the sound-transforming mechanism to provide a mobile connection from the tympanic membrane through an aerated middle ear space to the perilymph (2). The purpose of modern OCR is to obtain improved hearing, especially for conversational speech by restoring the stable sound transfer mechanism, which is achieved by coupling the tympanic membrane with a mobile stapes footplate via a reconstructed ossicular chain (3). The treatment of conductive hearing loss of various aetiology, due to neuroendocrine dysfunctions during pregnancy and post-partum (4), is currently based on replacing the affected ossicles with ossicular prosthesis in the attempt to give the patient better functional results and a higher level of social integration. The integrity of the auditory system is one of the prerequisites for the acquisition and the proper development of oral language and a person suffering from hypoacusis is more likely to have poorer professional results than their colleagues, will be less competitive on the labour market and will have smaller chances to complete higher education (5-7).

The term histological results refers to integration of the implant or rejection by the receiving organism. Long term defines a period of more than 5 years after implantation, but for the present study the time span was prolonged to 10 years or even longer. Synthetic or artificial bio-materials are those materials that are purposely introduced inside the human body in order to replace an organ, a tissue or a specific function.

Ossicular reconstruction materials are categorized as autografts, homografts, and alloplastic prosthetics. Each of these materials has advantages and disadvantages for prosthesis use (1).

Hydroxyapatite (HA), which is the mineral matrix of living bone, was introduced for OCR in 1984 by Grote (calcium phosphate ceramic) (8). It is a bioactive material that can achieve integration with surrounding bone and tissue. For instance, a collagen-hydroxyapatite composite material, characterized by a strong interaction between the collagen fibers and the hydroxyapatite crystals, can be successfully used as a bone substitute (9). In order to overcome the disadvantage of brittleness which makes HA technically difficult to sculpt, various composite materials that include HA were identified (silastic or polyethylene). 


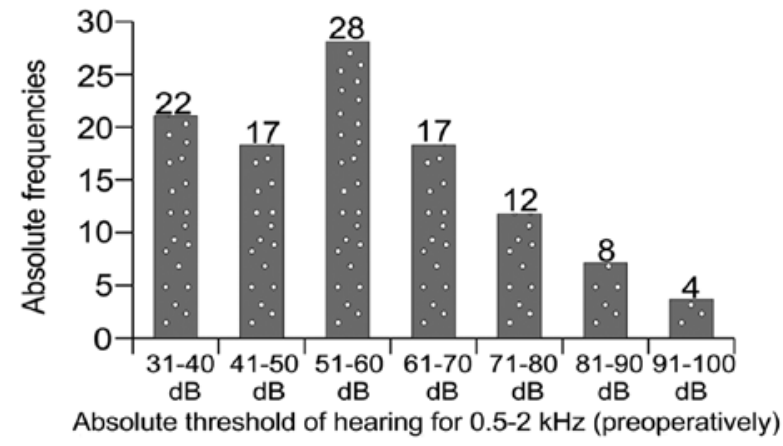

Figure 1. Preoperative absolute threshold of hearing for frequencies of $0.5-2 \mathrm{kHz}$.

Ceramic implants are biocompatible and react with surrounding tissues and bone and were used, with various results, worldwide. In the former communist bloc, including Romania, bio-ceramic implants were extensively used, throughout the 1990s and even well into the 2000s due to economic reasons. The advantage of ceramic implants is that they are not as harsh and can be placed in direct contact with the TM, without interposing cartilage $(10,11)$. However, its brittle nature made it difficult to handle and shape $(10,11)$.

Bio-vitro-ceramic PAW-1 is a solid, bioreactive, synthetic biomaterial, comprising fluro-hydroxyapatite and wollastonite microcrystals encompassed into a vitreous mass (glass); the material is obtained by controlled crystalization of a glass from the Silicium-Calcium-Magnesium-Phosphorus system with minute additions of Borum trioxyde and molecular fluoride. It represents a locally developed product (12). Bio-materials, both bio-reactive or bio-active are those that react physically or chemically to water solutions, cells and tissues of the recipient organism, creating physical and chemical bounds with these. A direct integration into the recipient structures results.

There are four main classes of synthetic bio-materials: Bio-tolerated (noble metals, alloys, plastics); bio-inert (carbon fibres, frialit, corindon, ruby, inert ceramic); bio-active (bio-glass, bio-ceramic, bio-vitro-ceramic, hydrogels, microspheres); composite bio-materials (association of two or three bio-materials from different classes).

\section{Materials and methods}

General. A retrospective non-controlled study was conducted by making a random selection of 200 long-term patients. The basic statistical criterion for the selection was post-operative time span. Of the 200 patients, 108 patients with both radical and partial mastoidectomy were included. OCR was performed in a 3-year period (1993-1996). Synthetic prosthetics PAW1 of autochthonous origin were used. Data analysis began in 2004 giving a mean follow-up period of 9.12 years, thereby allowing it to be considered for long-term evaluation. All patients were clinically evaluated (microscope otoscopy), both before and after the surgery, as follows: During the first 6 months, every month; for the next 6 months, every 2 months; in the 2nd year, every 6 months; for the next 2 years, every 8 months; starting from the 5 th year, every $12-16$ months; at any other time when an otorrhea episode occurred.

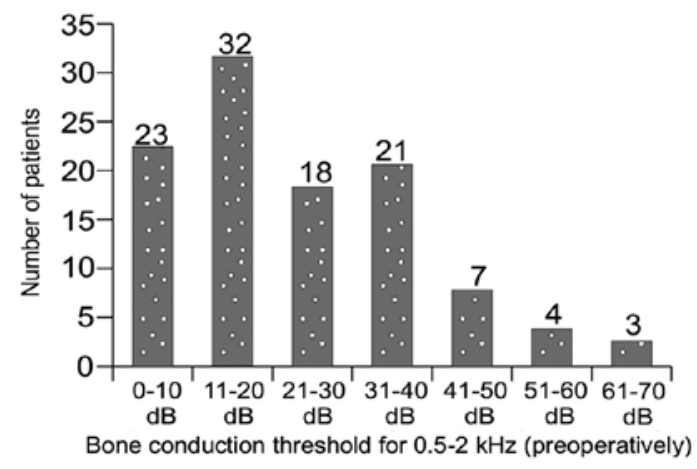

Figure 2. Preoperative threshold of bone conduction for frequencies of $0.5-2 \mathrm{kHz}$.

Absolute threshold. Absolute measurement of the absolute threshold of hearing and absolute threshold of bone conduction (between 0.5 and $2 \mathrm{kHz}$ ) was performed and used as a statistical indicator for rejection of prosthesis (Figs. 1 and 2). All ossicular reconstructions were performed at the end of a partial or radical mastoidectomy. The entire cohort had a long-term follow-up, as follows: 7 years $(n=1), 8$ years $(n=25)$, 9 years $(n=38), 10$ years $(n=38)$, and 11 years $(n=6)$. The types of prosthesis used, the absolute and relative frequencies of use and the rejection rate for each type of prosthesis are presented in Figs. 3 and 4. The type of prosthesis was also correlated to the intraoperative status of the ossicular chain (malleus, incus, stapes) as shown in Figs. 5-7.

Statistical analysis. Data were analyzed using SPSS ver.15. Measurement data were assessed as percentage, mean, or standard deviation (SD). Parametric tests (Student's t-test) or non-parametric tests (Mann-Whitney) were also applied. $\mathrm{P}$

\section{Results and Discussion}

The distribution of absolute and relative frequencies of rejection moments after the surgery are presented in Figs. 8 and 9. The rejection rate after 9.12 years was $21 \%(n=23)$. Histological integration rate was $79 \%(\mathrm{n}=85)$.

Over $50 \%$ of all rejections occur in short time span of one month post-operatively and the bulk of the rejections (74\%) occur during the first year. Although ejections no longer occur after 2 years after the surgical intervention, we found them even after a 7-year period. Insufficient follow-up may explain this result and allows us to define the rejection rate as an exponentially decreasing function (Fig. 10).

The success rate of the mastoidectomy (performed simultaneously with the implantation) is congruent to the rate of the successful implants (83 successful vs. 25 unsuccessful). A similar rate of success regarding mastoidectomy was recorded for the entire initial cohort of 200 patients: $77.5 \%$ success (155 patients) vs. 22.5\% failure (45 patients).

Our main aim was to define the situations and factors that influence the ossiculoplasty results and could yield a type of histological prognosis.

Based on the selection criteria of the studied group and by performing statistical analysis of the correlation of histological results and statistically significant variables we can formulate 


\begin{tabular}{|c|c|c|c|c|c|}
\hline $\begin{array}{l}\text { Type of } \\
\text { prosthesis }\end{array}$ & $\begin{array}{l}\text { Schematics } \\
\text { of prosthesis }\end{array}$ & $\begin{array}{l}\text { Schematics of } \\
\text { implantation }\end{array}$ & $\begin{array}{l}\text { Nr. of } \\
\text { patients - } \\
\text { absolute } \\
\text { frequency } \\
\text { of } \\
\text { implanta- } \\
\text { tion }\end{array}$ & $\begin{array}{l}\text { Relative } \\
\text { frequency of } \\
\text { implantation }\end{array}$ & $\begin{array}{l}\text { Rejection } \\
\text { rate }\end{array}$ \\
\hline $\begin{array}{l}\text { Lenticulo - } \\
\text { platino-pexy }\end{array}$ & & & 1 & $1 \%$ & $0 \%$ \\
\hline $\begin{array}{l}\text { Incudo- } \\
\text { stapedio- } \\
\text { pexy }\end{array}$ & & & 4 & $4 \%$ & $0 \%$ \\
\hline $\begin{array}{l}\text { Maleo- } \\
\text { vestibulo- } \\
\text { pexy }\end{array}$ & & & 5 & $5 \%$ & $0 \%$ \\
\hline $\begin{array}{l}\text { Miringo- } \\
\text { vestibulo- } \\
\text { pexy }\end{array}$ & & & 6 & $6 \%$ & $17 \%$ \\
\hline
\end{tabular}

Figure 3. The types of prosthesis used, the absolute and relative frequencies of use and the rejection rate for each type of prosthesis: Part 1.

pertinent conclusions regarding the implantation success of bioceramic materials.

Those variables, in regard to the time variable are structured as follows:

Before surgery variables. Age group: 11-20 years $(n=27)$, 21-30 years $(n=29), 31-40$ years $(n=22), 41-50$ years $(n=22)$, 51-60 years $(n=7), 61-70$ years $(n=1)$ (Fig. 16); clinical stage of disease: complicated $(n=10)$, not complicated $(n=98)$ (data not shown); type of disease: cholesteatoma $(n=75)$, non-cholesteatoma ( $\mathrm{n}=33$ ) (Fig. 11); type of tympanic membrane perforation: marginal $(n=38)$, central $(n=70)$ (Fig. 12); surgery rank: first intervention $(n=83)$, re-intervention $(n=20)$, 2nd re-intervention $(n=5)$ (Fig. 17).
Intraoperative data. Type of mastoidectomy: modified radical $(\mathrm{n}=27)$, partial ( $\mathrm{n}=81)$ (Fig. 18); Stapes status: footplate lysis $(n=11)$, footplate present and mobile $(n=12)$, footplate present and fixed $(n=13)$, stapes present and mobile $(n=36)$ (Fig. 13), stapes present and fixed $(n=36)$; Incus status: present and mobile $(n=2)$, bipolar lysis $(n=13)$, absent $(n=34)$, descendent lysis $(n=59)$ (Fig. 14); Malleus status: present and mobile $(n=76)$, present and fixed $(n=1)$, malleus mallei lysis $(n=3)$, malleus head lysis $(n=9)$, absent $(n=19)($ Fig. 15); Total lesional score: $0(n=79), 1-10(n=20), 11-20(n=4), 21-30(n=4), 41-50$ $(n=1)$ (data not shown); Type of prosthesis $(3,4,25)$.

Follow-up data. Period for complete epithelisation of cavity (Fig. 23): 2, 4, 6, 8, 10 and 12 months; Cavity self-cleansing: 


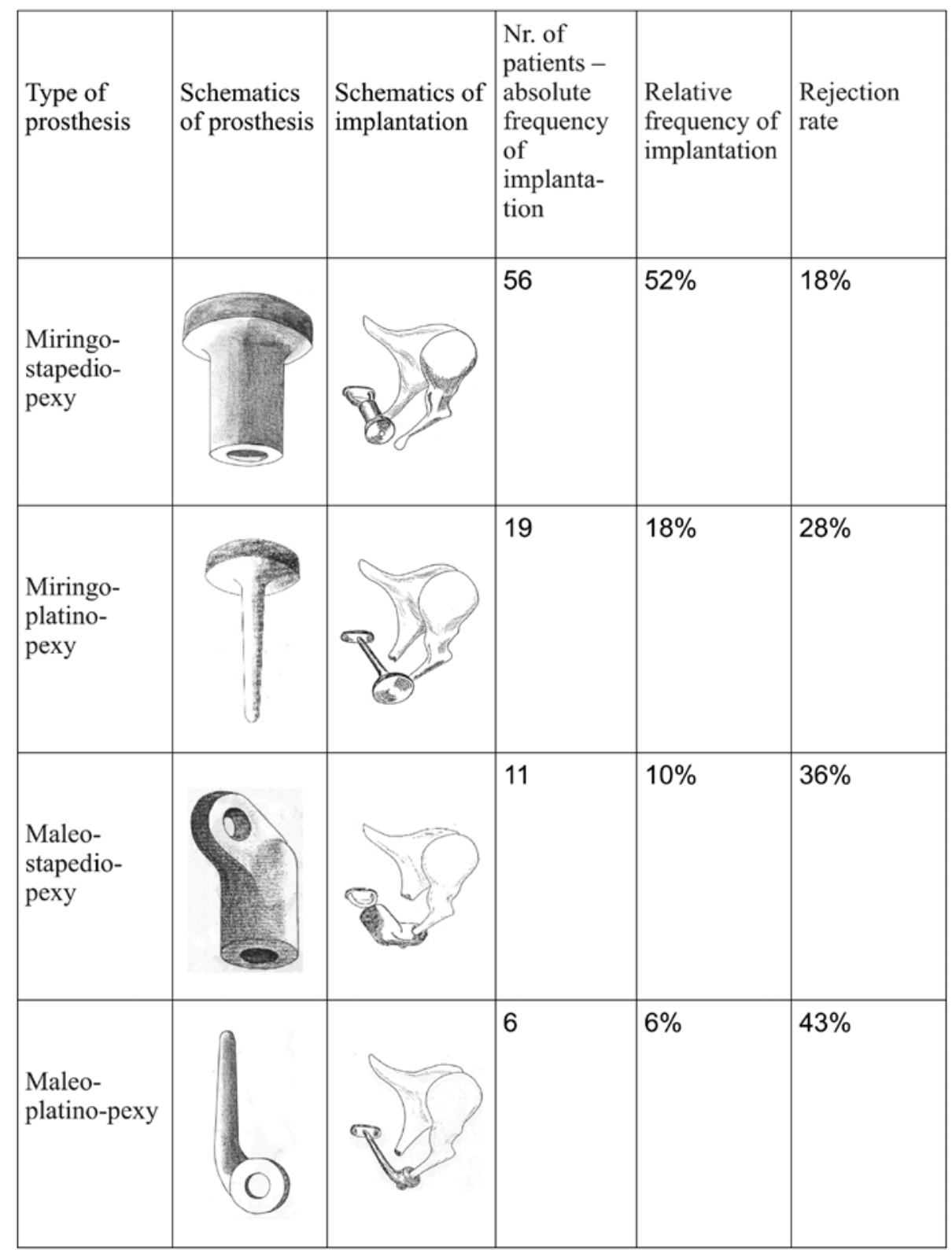

Figure 4. The types of prosthesis used, the absolute and relative frequencies of use and the rejection rate for each type of prosthesis: Part 2.

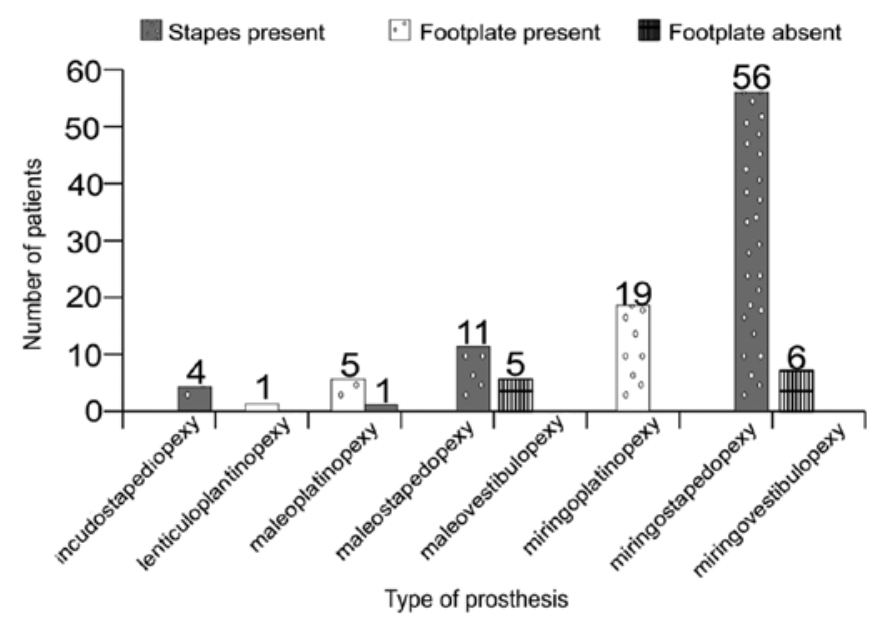

Figure 5. Absolute frequencies of the types of prosthesis used, in relation to the state of the stapes (type on ossicular chain interruption at the vestibular end). present or absent (Fig. 24); Mastoidectomy result (dainage effect): failure or success (Fig. 22).

Bio ceramics were developed as a solution to improve alloplastic biocompatibility. HA has many of the ideal characteristics required to be a good prosthesis. It is extremely biocompatible, exhibits a very low extrusion rate, has no transmittal of disease, and provides good sound transmission (13). HA forms a chemical bond with living bone and shows little biodegradation (14). It can also be placed in direct contact to the tympanic membrane or cartilage struts.

The general factors involved in OCR failure are: Problems with the design and function of the prosthesis, middle ear disease (mastoiditis) or Eustachian tube dysfunction. Each of these conditions eventually results in poor contact between the footplate and the graft (15). Although an optimally positioned prosthesis can migrate postoperatively and dislocate from the malleus handle, TM or stapes, the surgeon should provide the prerequisite for an optimal hearing outcome by placing 


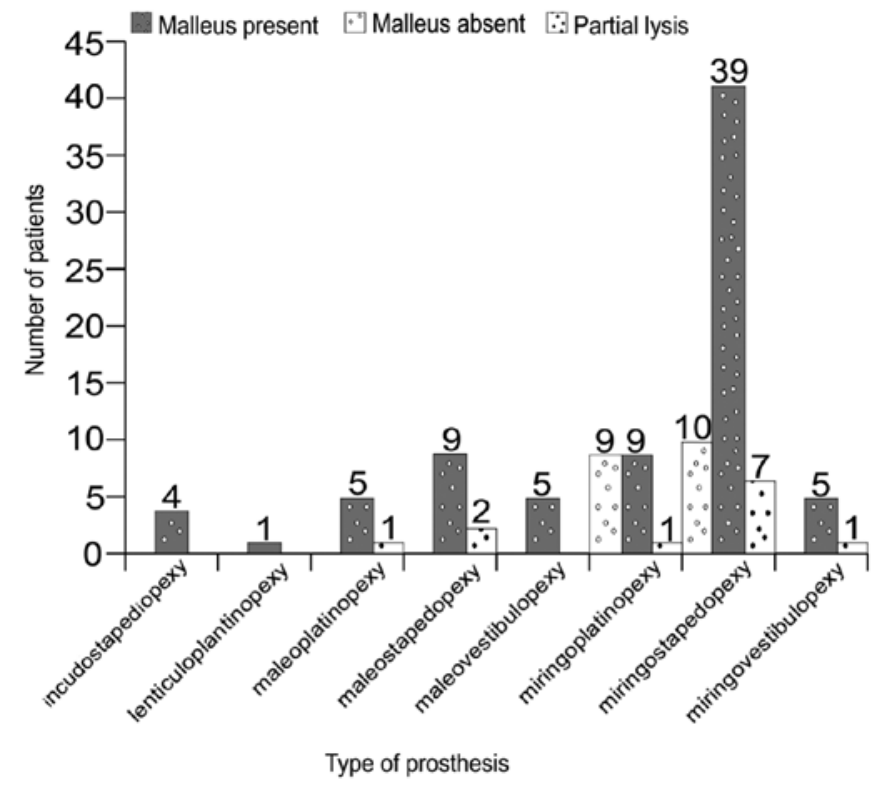

Figure 6. Absolute frequencies of types of prosthesis used, in relation to the state of the malleus.

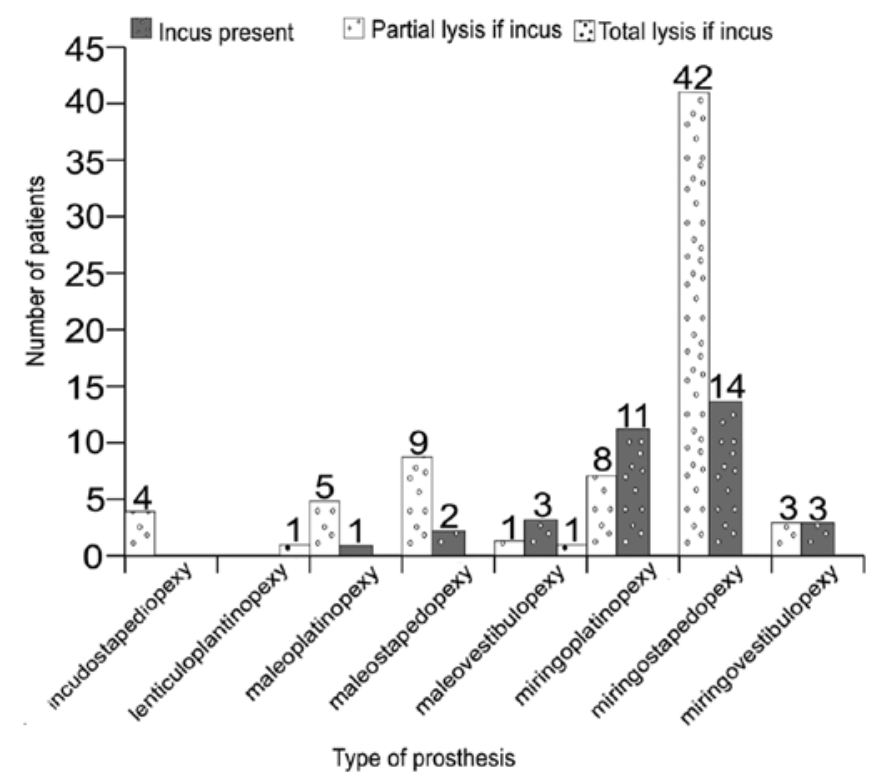

Figure 7. Absolute frequencies of types of prosthesis used, in relation to the state of the incus.

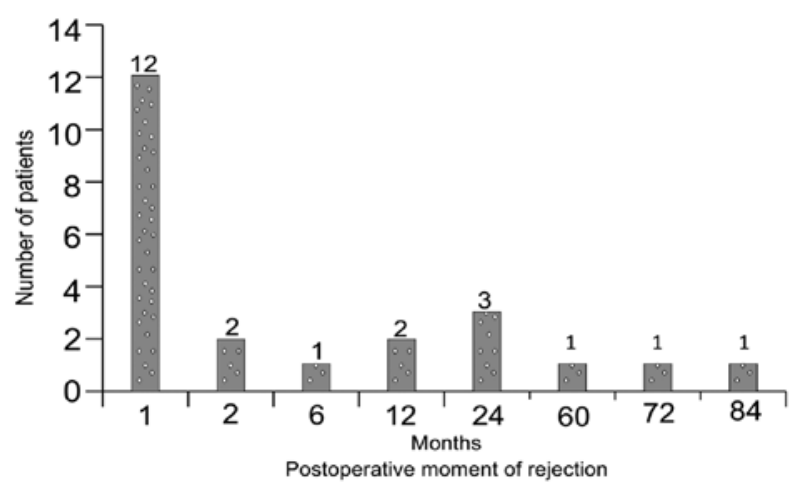

Figure 8. Distribution of absolute frequencies of the postoperative moment of implant rejection.

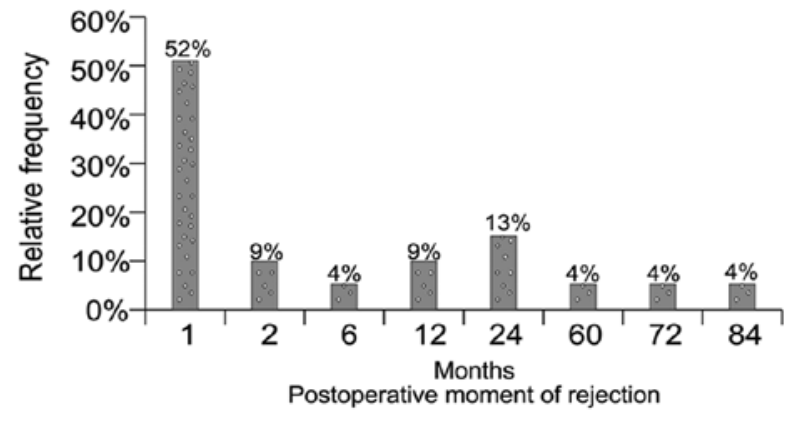

Figure 9. Distribution of relative frequencies of the postoperative moment of implant rejection.

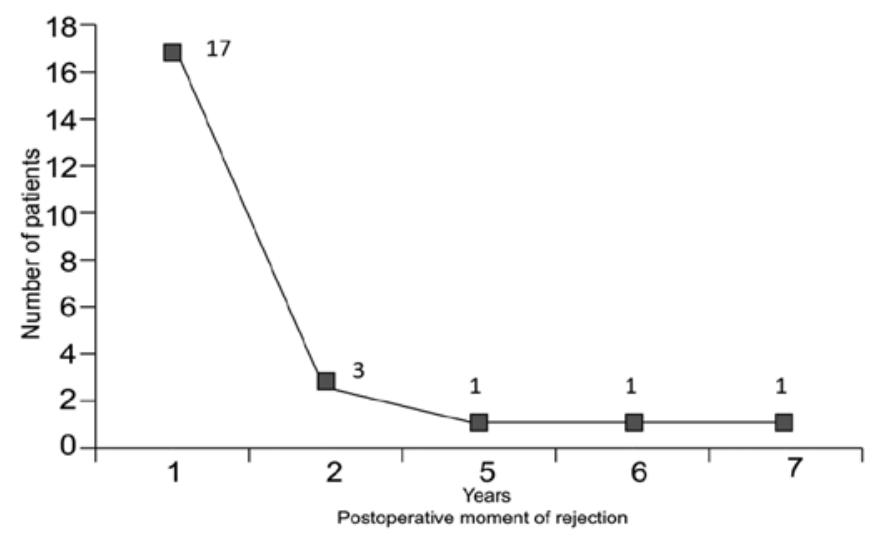

Figure 10. Distribution of absolute frequencies of the postoperative moment of implant rejection, as an exponentially decreasing function.

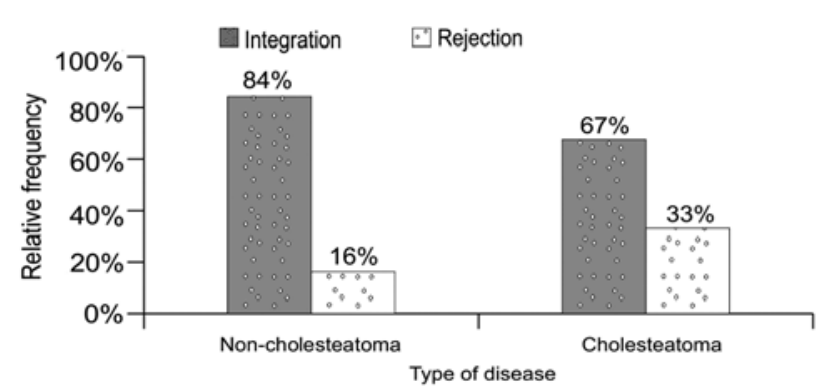

Figure 11. Influence of the type of disease (cholesteatoma) on implantation results.

the prosthesis properly (16). Perforation of the tympanic membrane, with or without extrusion of the prosthesis, may also occur (17). We found the influence of the clinical stage of the disease (complicated or non-complicated) to be paradoxal. A pertinent explanation for this is not readilly present and we tend to dismiss it as prognostic criteria.

The type of disease correlates to the expectations that the presence of cholesteatoma is a negative prognostic factor in general (Fig. 11). The type of tympanic perforation influences the rejection rate by $4 \%$ (Fig. 12). Eustachian tube dysfunction can favorize graft retraction and increases tension against the prosthesis therefore causing tympanic membrane perforation and prosthesis extrusion. Sustained tension may break the prosthesis or result in partial or complete extrusion. 


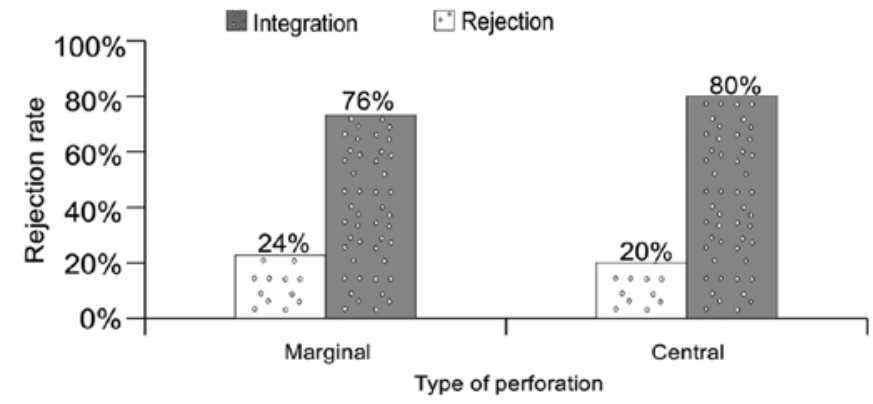

Figure 12. Influence of the type of tympanic perforation on implantation results.

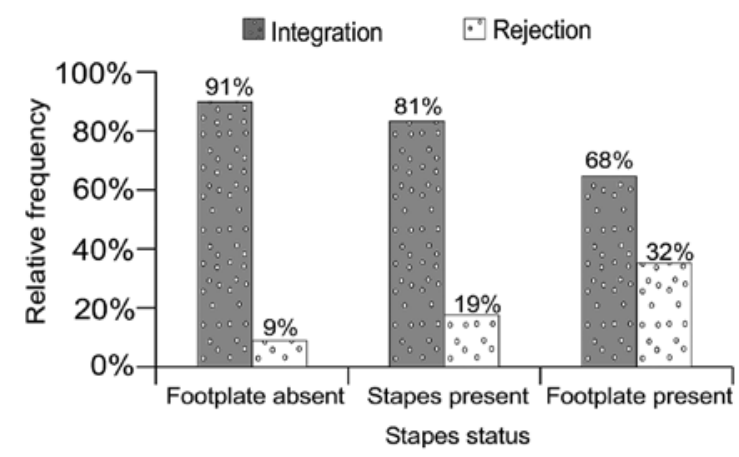

Figure 13. Influence of the stapes status on implantation results.

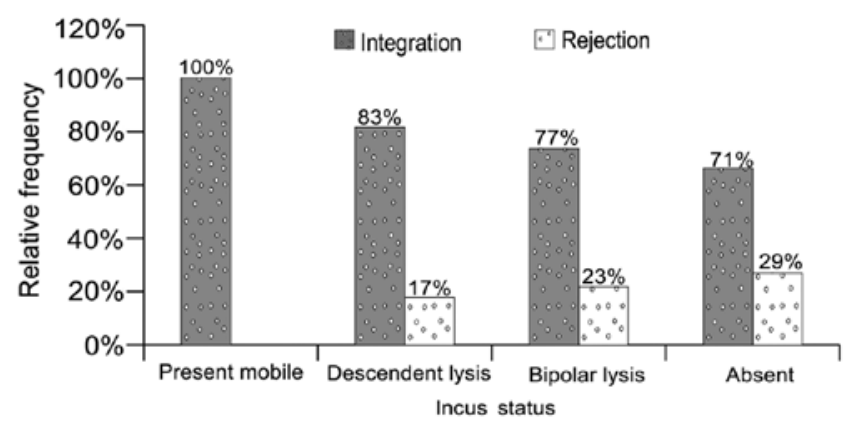

Figure 14. Influence of the incus status on implantation results.

It is difficult to interpret the status of the stapes as a factor on the rejection rate (Fig. 13). On the other hand, the status of the incus, as found intraoperatively, has predictive significance as seen in Fig. 14, which means that there is a direct causal relationship defined by an analitical function. The status of the malleus has a comparable predictive significance but does not have the same mathematical accuracy as the one mentioned above (Fig. 15).

Austin defines four groups in which the incus had been partially or completely eroded to emphasize the importance of malleus handle and stapes superstructure presence for OCR (18): A, malleus handle and stapes superstructure present (60\% occurrence); B, malleus handle present, stapes superstructure absent (23\%); C, malleus handle absent, stapes superstructure present (8\%); D, malleus handle and stapes superstructure absent $(8 \%)$.

As early as 1973, Bellucci noted a relationship between the OCR results and middle-ear status (19).

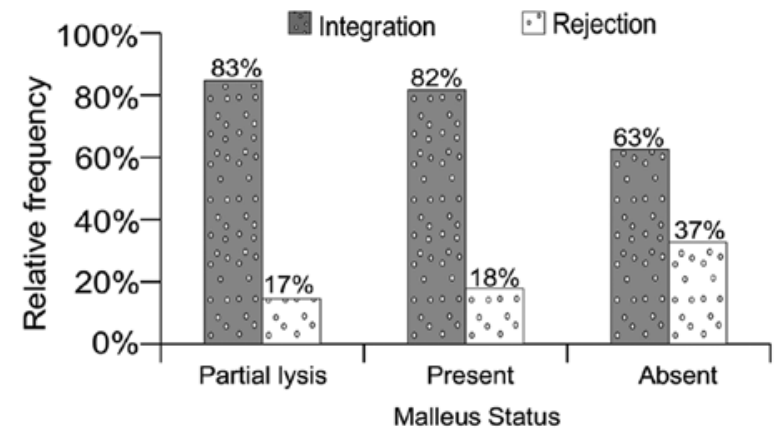

Figure 15. Influence of the malleus' status on implantation results.

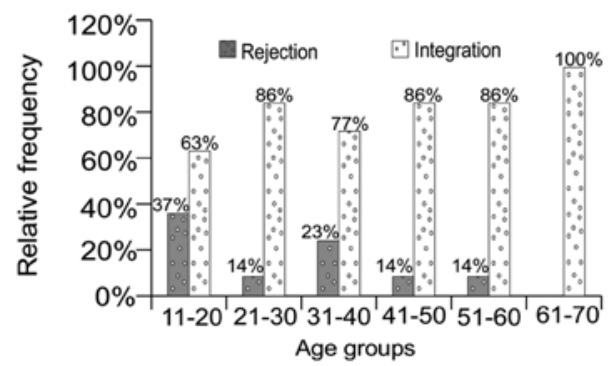

Figure 16. Influence of age groups on implantation results.

Kartush devised a scoring system called the middle ear risk index (MERI) to determine the probability of success in hearing restoration surgery (20-22). It takes into consideration the various stages of middle ear disease and ossicular status, including Austin's: otorrhea, perforation, cholesteatoma, middle ear granulation or effusion, previous surgery.

Black proposed a new evaluation system called SPITE for preoperative predictive factors of poor outcome (23): $\mathrm{S}$, surgical-complexity of the surgery; necessity of scutum and tympanic membrane repair; $\mathrm{P}$, prosthetic-absence of malleus or stapes; presentation of a $50 \mathrm{~dB}$ air bone gap; I, infection-chronic otorrhea; myringitis; $\mathrm{T}$, tissue-poor general condition of tissue, referring to extremes of youth (under 5 years of age) or advanced age (over 70 years); meatoplasty required; poor mucosa of the middle ear; E, eustachian tube dysfunction/middle ear effusion present; severely collapsed tympanic membrane.

Loss of the stapes superstructure was found by several researchers to be associated with poorer outcome in ossiculoplasty $(24,25)$.

Dornhoffer and Gardner suggested the ossicular outcomes parameters staging (OOPS): middle ear factors (drainage, mucosa ossicles); surgical factors (type of surgery, rank of surgery) (26).

Grote used for the first time referred to HA as OCR material with excellent long-term hearing results (8).

Goldenberg's study from 1992 reported low extrusion rates for hydroxyapatite (2.6\%) and plastipore (6.5\%) (27).

Numerous other studies have reported results of ossiculoplasty with HA or HAPEX prosthesis, with similar rejection results as our findings. Pasha et al studied 33 hydroxyapatite PORPs and TORPs. Hearing results were evaluated by postoperative mean ABG. The use of incus struts provided the 


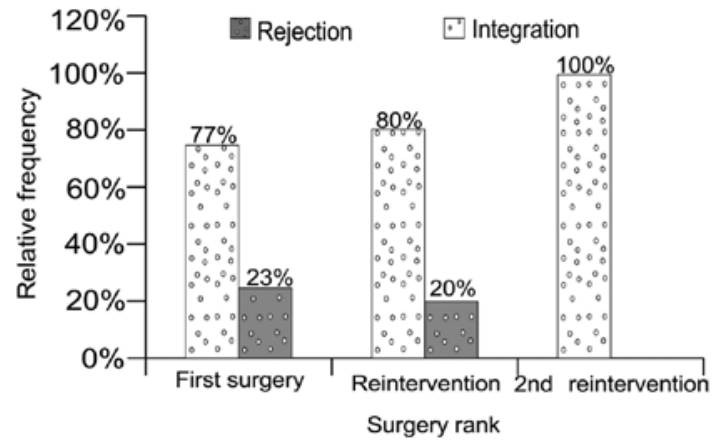

Figure 17. Influence of the rank of surgery on implantation results.

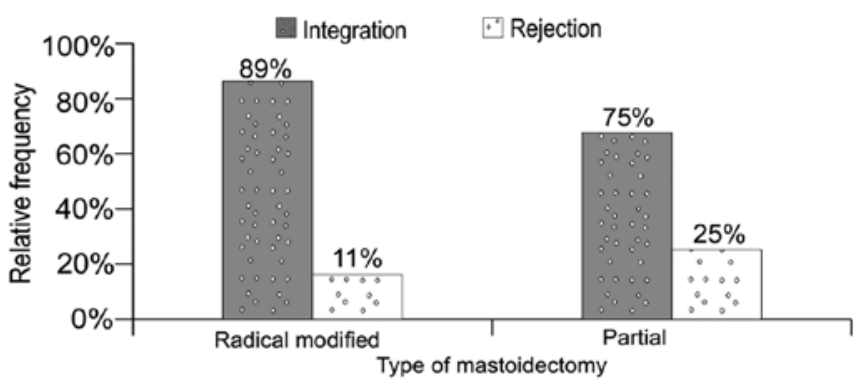

Figure 18. Influence of the type of surgery on implantation results.

best functional results. These patients had lower MERI scores in general and malleus handle present. In addition, PORPs extruded vs. no incus struts or TORPs (28).

House and Teufert, after an extensive study of 1,210 OCRs with HA and plastipore total ossicular replacement prostheses (TORPs) or partial ossicular replacement prostheses (PORPs) reported $63 \%$ implant integration. Hearing results were improved for first surgery patients and no chronic otitis media, when a cartilage graft was used, and for Plasti-Pore rather than hydroxyapatite (29).

Iurato et al (30), in an extensive review of the literature, investigates results of OCR in Austin-Kartush type A patients. After a minimum follow up of 12 months, incus interposition showed an $84 \%$ success rate vs. $82 \%$ for allograft (ceramics or HA) PORP.

Rondini-Gilli et al reported 100 patients with HA PORP $(n=65)$ or TORP $(n=35)$. Ten percent $(10 \%)$ of cases presented extrusion or displacement of the implants, more commonly when no cartilage cap was placed (31).

Dornhoffer and Gardner reconstructed 200 ears with HAPEX PORP or TORP and concluded that the significant prognostic factors were: The state of the ossicular chain, the state of the middle ear mucosa, presence of otorrhea, previous mastoidectomy, and revision surgery (25). Other authors compared the results of HA and titanium implants.

Truy et al (32) published a success rate of 55\% for HA TORP, $51 \%$ for Titanium TORP, $67 \%$ for HA PORP, $72 \%$ for Titanium PORP in a retrospective comparison of hydroxyapatite vs. titanium TORP and PORP.

Coffey et al conducted a study on 105 cases $(80$ with titanium and 25 with non-titanium implants) and reported a success rate of $50 \%$ for non-titanium cases and $77.1 \%$ for

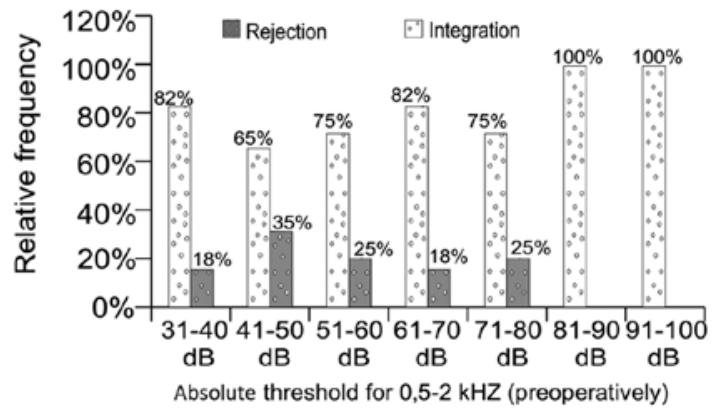

Figure 19. Influence of the preoperative absolute threshold of hearing on implantation results.

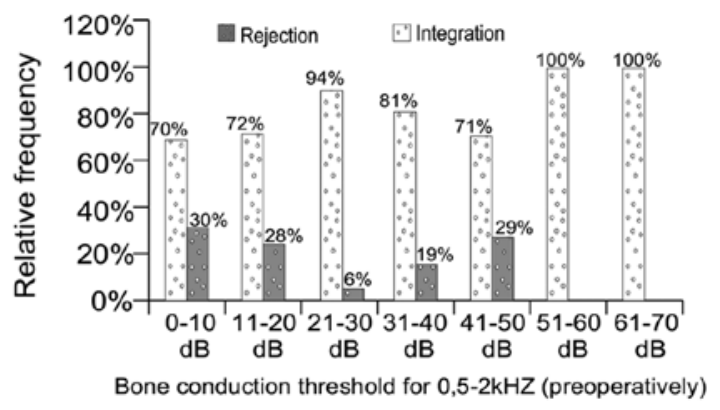

Figure 20. Influence of the preoperative threshold of bone conduction on implantation results.

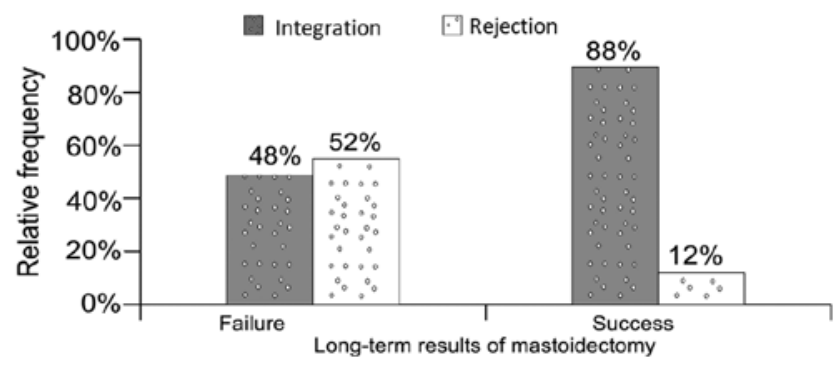

Figure 21. Influence of long-term results of the mastoidectomy on implantation results.

titanium cases; extrusion was observed with two non-titanium prostheses $(8.0 \%)$ and three titanium prostheses $(3.8 \%)(20,33)$.

Gardner $e t a l$, in a retrospective study comparing titanium reconstructions to non-titanium reconstructions, reported the successful rehabilitation of conductive hearing loss in $70 \%$ of PORPs vs. $44 \%$ of TORPs when titanium prostheses were used as compared to 48 and $21 \%$ of non-titanium-based partial and total reconstructions (34).

Emir et al (35) reviewed 304 cases of ossiculoplasty with intact canal wall. The success rate for autologous incus interposition was 58 vs. $56 \%$ for plastipore PORPs, while $9.3 \%$ of implants extruded.

We should also note that numerous studies reported similar rejection rates for the modern and largely used titanium implants.

Martin and Harner (36) reviewed 68 cases of OCR using a titanium TORP or PORP. Closure of the ABG to within $20 \mathrm{~dB}$ was obtained in $57 \%$. 


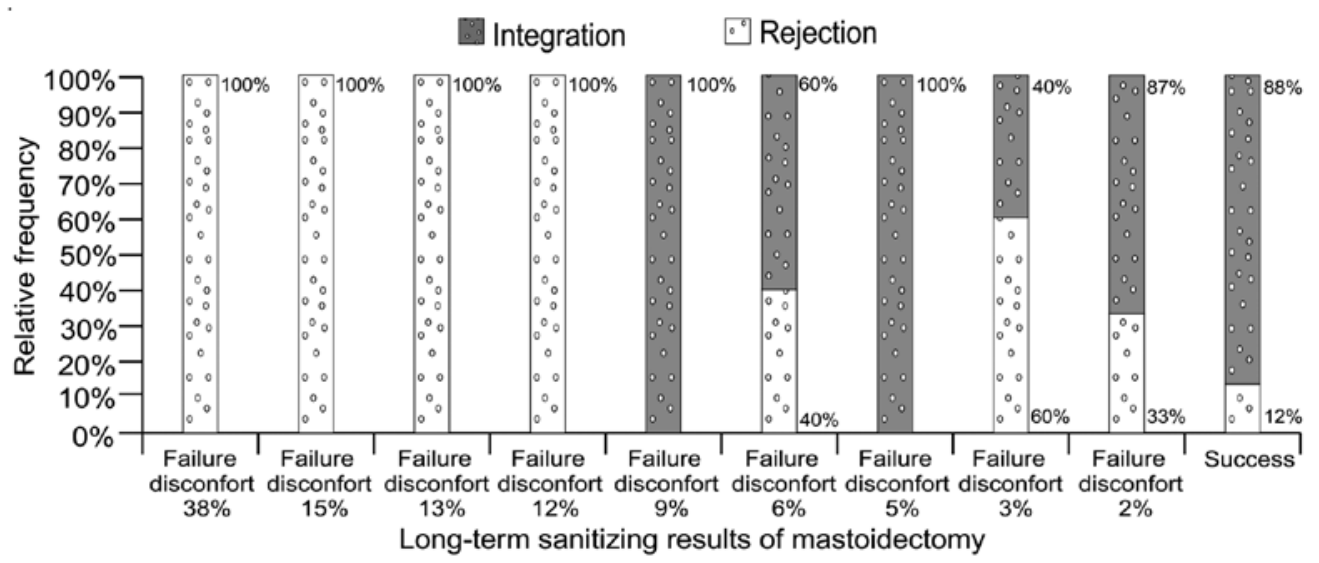

Figure 22. Influence of long-term drainage results of the mastoidectomy on implantation results.

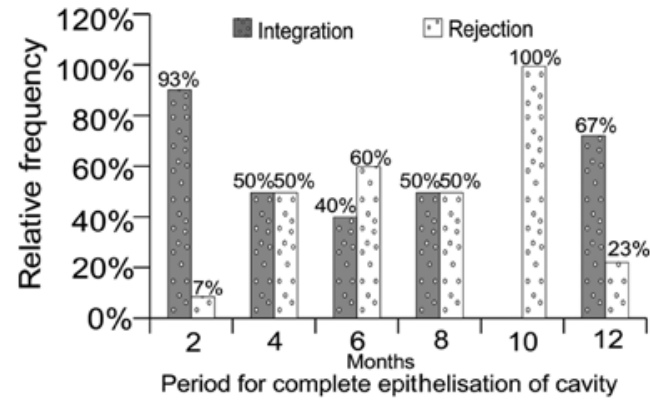

Figure 23. Influence of epithelisation period of cavity on implantation results.

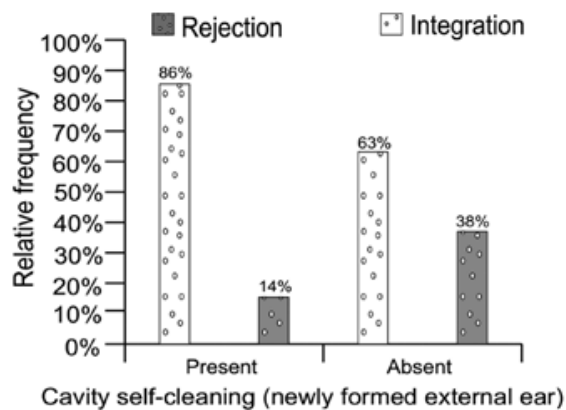

Figure 24. Influence of the presence of self-cleaning of the cavity (newly formed external ear) on implantation results.

Dalchow et al published results from 1,304 implanted titanium TORPs and PORPs. His overall success rate was $76 \%$ (37).

Neff et al (38) studied results for 18 OCR with titanium TORP and reported an $89 \%$ surgical success rate for an average follow-up time of 8 months.

De Vos et al (39) reported a success rate of $60 \%$ for both PORP and TORP on 149 ears, all implanted with titanium prosthesis. The extrusion rate was $3.5 \%$ and displacement of the prosthesis occurred in $4.3 \%$.

Vassbotn et al (40) published a report on 73 OCR with titanium prostheses (38 PORPs vs. 35 TORPs). After a mean follow up of 14 months, the success rate was $77 \%$ for the entire cohort.

The influence of the age group on the rejection rate was direct and analytic (Fig. 16). In other words, the rejection rate

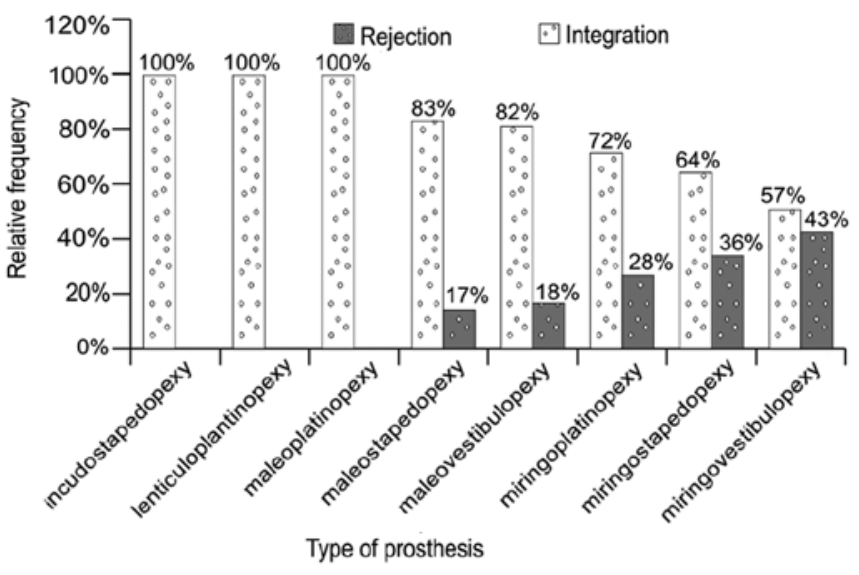

Figure 25. Influence of the types of prosthesis on implantation results.

was determined by an analytical function of the gravity of the disease which is inversly proportional to the patient's age and directly proportional to the degree of the hearing loss.

The rank of the surgery is also paradoxal in influencing the rejection rate, since a superior rank means better mastoid drainage and elimination of leassions and therefore better premises for histologic integration of implants (Fig. 17). The same discussion could be applied when discussing the type of surgery: Partial surgery increased the chance for remanent lessions (Fig. 18).

The total lesional score refers to all lesions found pre-, intra- and post-surgery, except those of the middlea ear mucosa. This score is therefore insignificant for the rejection rate and we should also consider that medical research can sometimes require a high degree of abstraction (41).

The pre-surgery absolute threshold of hearing and threshold of BC do not seem to have a statistical effect on the rejection rate (Figs. 19 and 20). Notably, the value of 6\% rejection rate for $\mathrm{BC}$ threshold between $21-30 \mathrm{~dB}$ is not consistent with the other results that are closer to $30 \%$ suggesting that, under certain conditions, some connection exists between these factors. A valid explanation for this discrepancy remains to be determined in future studies.

The long-term results of mastoidectomy have direct bearing on the results of implantation as shown in Figs. 21 and 22, as 
does the period of epithelisation after surgery and the rate of self-cleansing of the cavity (Figs. 23 and 24).

It is difficult to describe the influence of the type of prosthesis but we can nevertheless state that the smaller the part of ossicular chain replaced is, the smaller the rejection rate is (Figs. 3, 4 and 25). These findings are in accordance with the results from medical literature, which report improved functional results for PORP implantations as compared to TORP. Notably, the physical tension involved in all these interventions that have a high impact on the quality of life of the patient should be considered (42).

Ho et al reported achieving an air-bone gap lower than $20 \mathrm{~dB}$ in $64 \%$ of PORP and $45 \%$ of TORP implants (43).

Schmerber et al published a retrospective chart review of 111 OCRs with titanium prostheses. For PORP $77 \%$ of the cases were reported as successful vs. 52\% for TORP (44).

Vassbotn et al (40) reported $89 \%$ of PORP implants as successful vs. $63 \%$ of TORPs (29) while Siddiq and Raut, in a prospective study of 33 OCRs assessed the early results of titanium PORP and TORP in chronic ear disease and concluded that PORPs had a higher success rate $(85 \%)$ than TORPs (46\%) (45).

A large number of materials is available for reconstructing the ossicular chain. Therefore, the surgeon should assess the method and the type of prosthesis to be used very carefully in order to obtain the best hearing result with the smallest risk of complications. It is clear that optimal results depend, not only on the choice of material and design of the prosthesis, but also on the status of the middle ear in which it is placed, and also on the expertise of the surgeon and the techniques to be used.

Hydroxyapatite has many of the ideal characteristics required to be a good prosthesis with a high degree of biocompatibility, very low extrusion rate, low risk of disease transmission and good functional results. Of note, bioceramic implants are cheaper and can be produced locally, which is a great advantage for struggling economies.

The general factors that showed statistical influence in OCR failure are: Prior to surgery factors (age group, type of lesion-cholesteatoma, type of TM perforation); during surgery factors (type of surgery, state of incus, type of prosthesis); after surgery factors (results of mastoidectomy, period of complete epidermal growth, self-cleansing of cavity).

Additional causes of functional failure include improperly sized prosthesis (too short), sliding of the prosthesis, stapes anterior crural fracture, and contraction and movement of the healing tympanic membrane.

\section{Acknowledgements}

Not applicable.

\section{Funding}

No funding was received.

\section{Availability of data and materials}

Not applicable.

\section{Authors' contributions}

$\mathrm{HM}$ and MR are responsible for the original idea, conception, patient selection and care, operations, data collection and editing structure. MR is also responsible for first use of the implant material. HM and MR also contributed to design of prosthesis and final study. AIM and AMD gathered the medical information and reviewed the final version of the article. All authors read and approved the final manuscript.

\section{Ethics approval and consent to participate}

For this study, the agreement was obtained from the Research Ethics Committee of the Faculty of Medicine, Titu Maiorescu University.

\section{Patient consent for publication}

All patients provided informed consent and approved the publication of data.

\section{Competing interests}

The authors declare that they have no competing interests.

\section{References}

1. Bojrab DI and Babu SC: Ossiculoplasty I. In: Middle Ear and Mastoid Surgery. Habermann RSII (ed.) Thieme Verlag, New York-Stuttgart, pp151-158, 2004.

2. Hirsch BE: Ossicular chain reconstruction. In: Head and neck surgery. Myers E (ed.) Saunders Elsevier, pp1147-1162, 2008.

3. Colletti V, Carner M and Colletti L: TORP vs. round-window implant for hearing restoration of patients with extensive ossicular chain defect. Acta Otolaryngol 129: 449-452, 2009.

4. Trifu S, Vladuti A and Popescu A: Neuroendocrine aspects of pregnancy and postpartum depression. Acta Endocrinol (Bucharest) 15: 410-415, 2019.

5. Mocanu $\mathrm{H}$ and Oncioiu I: The influence of clinical and environmental risk factors in the etiology of congenital sensorineural hearing loss in the Romanian population. Iran J Publ Health 48: 2301-2303, 2019.

6. Mocanu H: The role of perinatal hearing screening in the normal development of the infant's language. In: Debating Globalization. Identity, Nation and Dialogue fourth Edition. Boldea I and Sigmirean C (eds). Arhipeleag XXI Press, Tirgu Mures, pp562-569, 2017.

7. Mocanu H: The economic impact of early diagnosis of congenital hearing loss. 4th International Conference Globalization, Intercultural Dialogue and National. Targu Mures, Romania.

8. Grote JJ: Tympanoplasty with calcium phosphate. Arch Otolaryngol 110: 197-199, 1984.

9. Ficai A, Andronescu E, Ghitulicã C, Voicu G, Trandafir V, Mânzu D, Ficai M and Pall S: Colagen/hydroxyapatite interactions in composite biomaterials. Mat Plast 46: 11-15, 2009.

10. Niparko JK, Kemink JL, Graham MD and Kartush JM: Bioactive glass ceramic in ossicular reconstruction: A preliminary report. Laryngoscope 98: 822-825, 1988.

11. McElveen JT, Cunningham CD and Sheehy JL: Ossicular reconstruction. In: Otologic Surgury. Saunders Elsevier, pp161-171, 2001.

12. Rădulescu M and Popescu-Negreanu T: Timpanoplastie cu biovitroceramica PAW-1. Oto-Rino-Laringologia XL: 97-102, 1995.

13. Bojrab DI, Causse JB, Battista RA, Vincent R, Gratacap B and Vandeventer G: Ossiculoplasty with composite prostheses. Overview and analysis. Otolaryngol Clin North Am 27: 759-776, 1994.

14. Hörman $\mathrm{K}$ and Donath $\mathrm{K}$ : Is hydroxylapatite ceramic an adequate biomaterial in ossicular reconstruction? Am J Otol 8: 402-409, 1987. 
15. Sellari-Franceschini S, Piragine F, Bruschini P and Berrettini S: TORPs and PORPs: Causes of failure. Am J Otol 8: 551-552, 1987.

16. Neudert M, Bornitz M, Mocanu H, Lasurashvili N, Beleites T, Offergeld $\mathrm{C}$ and Zahnert T: Feasibility study of a mechanical real-time feedback system for optimizing the sound transfer in the reconstructed middle ear. Otol Neurotol 39: e907-e920, 2018.

17. Batti JS and Bluestone CD: Ossiculoplasty. In: Surgical Atlas of Pediatric Otolaryngology. Bluestone CD and Rosenfeld R (eds). 2nd edition. BC Decker Inc, Hamilton, ON, pp75-89, 2002.

18. Austin DF: Ossicular reconstruction. Otolaryngol Clin North Am 5: 145-160, 1972.

19. Bellucci RJ: Dual classification of tympanoplasty. Laryngoscope 83: 1754-1758, 1973.

20. Kartush JM: Ossicular chain reconstruction: Capitulum to malleus. Otolaryngol Clin North Am 27: 689-715, 1994.

21. Simon $C$ and Makishima T: Ossicular reconstruction. In: Grand rounds presentation. The University of Texas Medical Branch, Department of Otolaryngology, 2009.

22. Trifu S: Neuroendocrine insights into burnout syndrome. Acta Endocrinol (Buchar) 15: 404-405, 2019.

23. Black B: Ossiculoplasty prognosis: The spite method of assessment. Am J Otol 13: 544-551, 1992.

24. Mills RP: The influence of pathological and technical variables on hearing results in ossiculoplasty. Clin Otolaryngol Allied Sci 18: 202-205, 1993

25. Smyth GD and Patterson CG: Results of middle ear reconstruction: Do patients and surgeons agree? Am J Otol 6 : $276-279,1985$

26. Dornhoffer JL and Gardner E: Prognostic factors in ossiculoplasty: A statistical staging system. Otol Neurotol 22: 299-304, 2001.

27. Goldenberg RA: Hydroxylapatite ossicular replacement prostheses: Results in 157 consecutive cases. Laryngoscope 102: 1091-1096, 1992.

28. Pasha R, Hill SL III and Burgio DL: Evaluation of hydroxyapatite ossicular chain prostheses. Otolaryngol Head Neck Surg 123 425-429, 2000

29. House JW and Teufert KB: Extrusion rates and hearing results in ossicular reconstruction. Otolaryngol Head Neck Surg 125: $135-141,2001$

30. Iurato S, Marioni G and Onofri M: Hearing results of ossiculoplasty in Austin-Kartush group A patients. Otol Neurotol 22: 140-144, 2001.

31. Rondini-Gilli E, Grayeli AB, Borges Crosara PF, El Garem H, Mosnier I, Bouccara D and Sterkers O: Ossiculoplasty with total hydroxylapatite prostheses anatomical and functional outcomes. Otol Neurotol 24: 543-547, 2003.
32. Truy E, Naiman AN, Pavillon C, Abedipour D, Lina-Granade G and Rabilloud M: Hydroxyapatite versus titanium ossiculoplasty. Otol Neurotol 28: 492-498, 2007.

33. Coffey CS, Lee FS and Lambert PR: Titanium versus nontitanium prostheses in ossiculoplasty. Laryngoscope 118: 1650-1658, 2008.

34. Gardner EK, Jackson CG and Kaylie DM: Results with titanium ossicular reconstruction prostheses. Laryngoscope 114: 65-70, 2004.

35. Emir H, Kizilkaya Kaptan Z, Göcmen H, Uzunkulaoglu H, Tuzuner A, Bayiz U and Samim E: Ossiculoplasty with intact stapes: Analysis of hearing results according to the middle ear risk index. Acta Otolaryngol 129: 1088-1094, 2009.

36. Martin AD and Harner SG: Ossicular reconstruction with titanium prosthesis. Laryngoscope 114: 61-64, 2004.

37. Dalchow CV, Grun D and Stupp HF: Reconstruction of the ossicular chain with titanium implants. Otolaryngol Head Neck Surg 125: 628-630, 2001.

38. Neff BA, Rizer FM, Schuring AG and Lippy WH: Tympano-ossiculoplasty utilizing the Spiggle and Theis titanium total ossicular replacement prosthesis. Laryngoscope 113: $1525-1529,2003$.

39. De Vos C, Gersdorff M and Gérard JM: Prognostic factors in ossiculoplasty. Otol Neurotol 28: 61-67, 2007.

40. Vassbotn FS, Møller P and Silvola J: Short-term results using Kurz titanium ossicular implants. Eur Arch Otorhinolaryngol 264: 21-25, 2007.

41. Alecu I, Mocanu H and Călin IE: Intellectual mobility in higher education system. Rom J Mil Med 120: 16-21, 2017.

42. Trifu S, Delcuescu C and Boer CM: Psychosomatics and psychical tension (clinical research). Procedia Soc Behav Sci 33: 128-132, 2012.

43. Ho SY, Battista RA and Wiet RJ: Early results with titanium ossicular implants. Otol Neurotol 24: 149-152, 2003.

44. Schmerber S, Troussier J, Dumas G, Lavieille JP and Nguyen DQ: Hearing results with the titanium ossicular replacement prostheses. Eur Arch Otorhinolaryngol 263: 347-354, 2006.

45. Siddiq MA and Raut VV: Early results of titanium ossiculoplasty using the Kurz titanium prosthesis-a UK perspective. J Laryngol Otol 121: 539-544, 2007.

(i) $\odot$ This work is licensed under a Creative Commons Attribution-NonCommercial-NoDerivatives 4.0 International (CC BY-NC-ND 4.0) License. 